\title{
Performances de reproduction et de production laitière des chèvres Draa au Maroc
}

\author{
I. Boujenane ${ }^{1 *}$ N. Lichir ${ }^{1}$ A. El Hazzab ${ }^{2}$
}

\begin{abstract}
Mots-clés
Caprin - Chèvre Draa Reproduction - Production laitière Intervalle entre parturitions Durée de lactation - Maroc.
\end{abstract}

\begin{abstract}
Résumé
Le principal objectif de cette étude a été une contribution à la connaissance des chèvres de race Draa. L'étude a porté sur l'analyse des performances de reproduction et de production laitière des chèvres Draa de la station de Skoura entre 1989 et 2001. L'âge à la première mise bas a été en moyenne de 25,5 mois. La durée de gravidité moyenne a été de 153 jours. L'intervalle entre deux mises bas et l'intervalle mise bas - saillie fécondante ont été respectivement de 157 et 206 jours. La durée de lactation a été de 133 jours. La production laitière a été de $0,46 \mathrm{~kg}$ par jour et de $61,3 \mathrm{~kg}$ par lactation totale. Il a été conclu qu'il serait intéressant d'exploiter la variabilité génétique qui existe au sein de la race à des fins de sélection.
\end{abstract}

\section{INTRODUCTION}

Le cheptel caprin au Maroc a été évalué à près de 5,3 millions de têtes (13). Il est caractérisé par son adaptation aux conditions du pays. Les caprins sont concentrés essentiellement dans les zones défavorisées de montagnes et de parcours dégradés, dans lesquelles ils constituent une activité économique importante pour la population. Le cheptel caprin est composé d'un petit nombre de races ou de populations locales hétérogènes dont les performances de reproduction et de production sont mal connues $(2,3)$. Parmi ces ressources génétiques caprines figure la race Draa, dont l'effectif avoisine 20000 têtes, qui est élevée en petits troupeaux, dans les oasis de la vallée de Draa, pour la production de lait et de viande.

Dans le présent travail, les données des chèvres Draa de la station de Skoura ont été analysées afin de préciser les performances de reproduction et de production laitière, et de jeter les premiers jalons d'un programme de sélection pour l'amélioration de la race.

\footnotetext{
1. Département de productions et de biotechnologies animales, Institut agronomique et vétérinaire Hassan II, BP 6202, Rabat-Instituts, 10101 Rabat, Maroc. Tél./fax : +212537776420

E-mail : i.boujenane@iav.ac.ma

2. Office régional de mise en valeur agricole d'Ouarzazate, Ouarzazate, Maroc.

* Auteur pour la correspondance
}

\section{MATERIEL ET METHODES}

\section{Présentation de la race Draa}

L'animal de race Draa est de format moyen à ossature fine. La hauteur au garrot est en moyenne de $66 \mathrm{~cm}$ et la longueur du corps varie entre 55 et $65 \mathrm{~cm}$. La tête est fine, triangulaire. Les oreilles petites et pointues sont légèrement orientées vers l'avant. L'encolure est mince et peu longue, et porte très souvent des pendeloques. Le tronc est caractérisé par un garrot non saillant, une poitrine profonde et une croupe inclinée portant une queue courte et dressée. La peau est fine, souple et porte des poils ras. Toutes les couleurs sont représentées au niveau des robes. La mamelle, relativement développée, est attachée vers l'avant et porte des trayons dirigés vers l'extérieur (figure 1).

\section{Milieu naturel de la station}

Les données analysées provenaient des chèvres Draa de la station de Skoura. Celle-ci est située à une quarantaine de kilomètres au sud-est d'Ouarzazate. Le climat dominant dans la région est de type continental présaharien avec un hiver froid à relativement frais et un été chaud. La pluviométrie moyenne calculée sur les vingt dernières années a été de $140 \mathrm{~mm}$, variant selon les années de 80 à $170 \mathrm{~mm}$. Les températures moyennes ont varié entre $2^{\circ} \mathrm{C}$ au mois de janvier et $39^{\circ} \mathrm{C}$ au mois de juillet. 

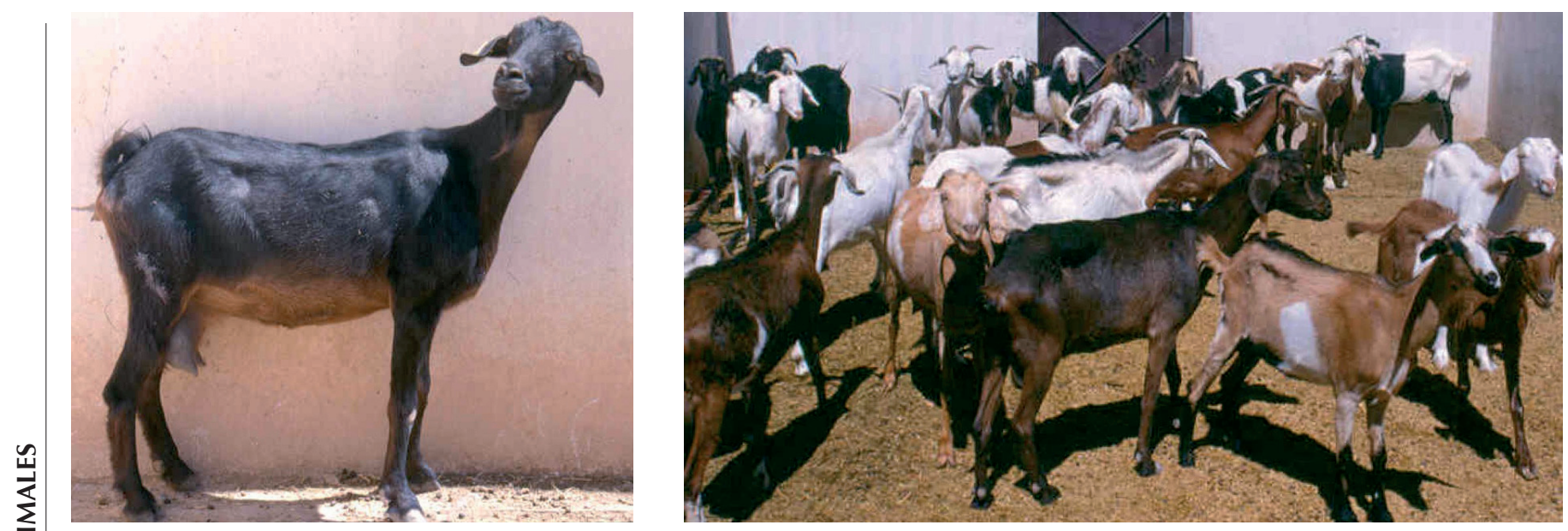

Figure 1 : chèvres Draa.

\section{Matériel animal et conduite du troupeau}

Les chèvres du troupeau ont été achetées en 1982 dans la vallée de Draa. L'effectif de départ était constitué de 80 chèvres et a varié selon les années entre 55 et 160 chèvres.

Le troupeau a été conduit en stabulation permanente dans une chèvrerie aménagée permettant la séparation des animaux selon le sexe, l'âge et l'état physiologique. Les lots ont été constitués de groupes de femelles sans lien de parenté avec les mâles. Le choix des boucs destinés à la reproduction a été basé sur leur vigueur et la production laitière de leur mère. Les chèvres ont été mises à la lutte deux à trois mois après la mise bas. Elles ont été séparées des boucs en fin de gravidité et en début de lactation.

Il y a eu deux périodes distinctes sur le plan alimentaire au niveau de la station. De 1982 à 1994, l'alimentation des chèvres a été à base de fourrage vert (luzerne), de foin de luzerne, de paille et de concentré (pulpe sèche de betterave, datte, orge, son et aliment composé). A partir de 1994, l'alimentation distribuée a été à base de foin de luzerne, de luzerne déshydratée, de son de blé, de dattes, d'aliment composé et de pulpe sèche de betterave.

En matière de prophylaxie, les animaux ont bénéficié régulièrement de vaccinations préventives contre l'entérotoxémie. Les actions curatives ont concerné le traitement des maladies parasitaires, des coccidioses et des mammites.

\section{Contrôles effectués}

Les fichiers de données qui ont été exploités comportaient les informations relatives :

- à la généalogie (numéro de la chèvre, numéro du père, numéro de la mère) et à la date de naissance ;

- aux contrôles laitiers mensuels (quantité de lait aux différents relevés mensuels et leurs dates) ;

- aux résultats de reproduction (date de saillie, date de mise bas, taille de la portée à la naissance et poids corporel à la mise bas).

Les lactations ont été réalisées par 293 chèvres Draa nées entre 1987 et 1999 et ayant mis bas entre 1988 et 2005. La traite a été effectuée manuellement deux fois par jour. La quantité de lait issue de chaque traite et de chaque chèvre a été pesée et enregistrée sur la fiche de lactation de la chèvre.

\section{Variables étudiées et analyses statistiques}

A partir des différents contrôles effectués, les variables suivantes ont été calculées : âge à la première mise bas, intervalle mise bas - saillie fécondante, durée de gravidité, intervalle mise bas - mise bas, durée de lactation, production laitière totale et production laitière journalière. La production laitière totale a été calculée à partir des contrôles laitiers mensuels en utilisant la méthode du test interval method (10).

Les données ont été analysées en utilisant les procédures Means et Freq du logiciel SAS (18) afin d'obtenir les statistiques descriptives et les distributions de fréquences des différentes variables étudiées.

\section{RESULTATS ET DISCUSSION}

\section{Répartition des lactations selon le rang de lactation et le mois de mise bas}

Le rang de lactation des chèvres Draa dans la station de Skoura a varié de 1 à 11 . Néanmoins, 55 p. 100 des chèvres étaient en première ou en deuxième lactation, alors que les lactations de rang 4 et plus n'ont représenté que 30 p. 100 de l'ensemble des données.

Les chèvres Draa ont eu des mises bas étalées tout au long de l'année. Toutefois, la fréquence des mises bas a été très élevée durant les mois de février, mars et avril avec respectivement $12,10,5$ et 12,2 p. 100, alors qu'elle a été faible en juin et septembre avec respectivement 4,7 et 3,1 p. 100. Cet étalement des mises bas sur toute l'année semble indiquer une activité sexuelle continue chez les chèvres Draa avec une légère diminution en hiver.

\section{Age à la première mise bas}

L'âge à la première mise bas des chèvres Draa a été en moyenne de 766 jours, soit 25,5 mois, avec un écart-type de 234 jours (tableau I). Les mises bas ayant eu lieu pour la première fois avant l'âge de 19 mois ont été rares et ont représenté 14,8 p. 100 de l'ensemble des mises bas. En revanche, celles ayant eu lieu après l'âge de 27 mois ont représenté plus de 32 p. 100 des mises bas (figure 2). Chez la même race, Hachi (8) a rapporté un âge de 10,7 mois chez les éleveurs chez qui la lutte n'était pas contrôlée et de 23 mois en station. Cet âge à la première mise bas tardif a été similaire à celui de 25 mois enregistré chez les chèvres Anglo-Nubiennes (6). En revanche, il a été supérieur à ceux des chèvres de races Alpine (6), Red Sokoto (9) et Maradi (14) qui ont été respectivement de 21, 14 et 13,6 mois. L'âge à la première mise bas des chèvres Draa a été également plus élevé que celui des chèvres locales coréennes dans les conditions naturelles (412,1 jours) ou intensives (382,0 jours) (19). 
L'âge à la première mise bas est un paramètre de reproduction qui renseigne sur la précocité des jeunes femelles à l'entrée en production. Sa variation peut être expliquée par la période de naissance de l'animal, le retard de la mise à la reproduction des jeunes chèvres, la cohabitation entre les boucs et les chèvres d'une façon permanente, et la conduite alimentaire des chèvres. Ces résultats ont montré clairement que des marges de progrès dans la conduite peuvent être réalisées durant la phase d'élevage des jeunes chèvres afin de mieux maîtriser leur croissance et d'avancer de quelques semaines leur âge à la première mise bas.

\section{Intervalle mise bas - saillie fécondante}

Chez les chèvres Draa, l'intervalle mise bas - saillie fécondante a été en moyenne de 206 jours, avec un écart-type de 138 jours (tableau I). Quelques chèvres (9,5 p. 100) ont réussi leur saillie fécondante durant les 90 jours qui ont suivi la précédente mise bas,

\section{Tableau I}

Nombre, moyenne arithmétique (MA), écart-type (ET) et coefficient de variation (CV) des performances zootechniques des chèvres Draa

\begin{tabular}{|c|c|c|c|c|}
\hline Paramètre & Nb. & MA & ET & CV (\%) \\
\hline $\begin{array}{l}\text { Age à la première } \\
\text { mise bas (jours) }\end{array}$ & 271 & 766 & 234 & 30,5 \\
\hline $\begin{array}{l}\text { Intervalle mise bas - } \\
\text { saillie fécondante (jours) }\end{array}$ & 74 & 206 & 138 & 70,0 \\
\hline Durée de gravidité (jours) & 79 & 153 & 1,34 & 0,88 \\
\hline $\begin{array}{l}\text { Intervalle mise bas - } \\
\text { mise bas (jours) }\end{array}$ & 555 & 357 & 157 & 44,0 \\
\hline $\begin{array}{l}\text { Taille de la portée } \\
\text { à la naissance }\end{array}$ & 841 & 1,40 & 0,53 & 37,9 \\
\hline $\begin{array}{l}\text { Poids corporel } \\
\text { à la mise bas (kg) }\end{array}$ & 792 & 31,3 & 6,38 & 20,4 \\
\hline Durée de lactation (jours) & 754 & 133 & 45,1 & 33,9 \\
\hline $\begin{array}{l}\text { Production laitière } \\
\text { totale }(\mathrm{kg})\end{array}$ & 709 & 61,3 & 23,8 & 38,8 \\
\hline $\begin{array}{l}\text { Production laitière } \\
\text { journalière }(\mathrm{kg})\end{array}$ & 2764 & 0,46 & 0,26 & 56,5 \\
\hline
\end{tabular}

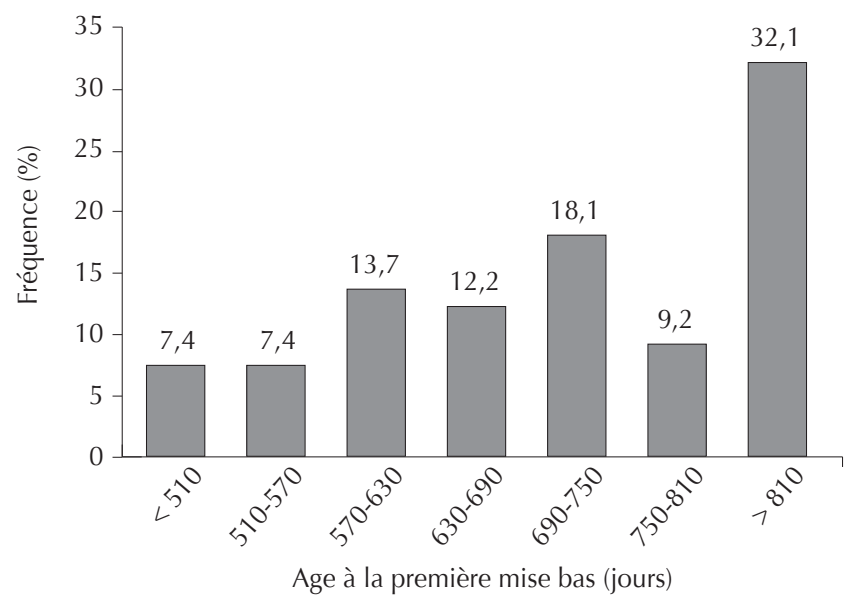

Figure 2 : distribution des fréquences de l'âge à la première mise bas des chèvres Draa $(n=271)$. tandis que presque 60 p. 100 d'entre elles ont été fécondées entre 90 et 210 jours, c'est-à-dire lors de la saison sexuelle suivante, et 17 p. 100 l'ont été 270 jours après la mise bas (figure 3). Chez les chèvres indiennes de différentes races, cet intervalle a été en moyenne de 205,2 jours (17).

L'allongement de l'intervalle mise bas - saillie fécondante est probablement la conséquence de la mise à la reproduction tardive des chèvres dans une stratégie de reproduction visant à concentrer les mises bas sur une courte période.

\section{Intervalle mise bas - mise bas}

L'intervalle entre deux mises bas successives chez les chèvres Draa a été en moyenne de 357 jours, soit 11,9 mois, avec un écarttype de 157 jours (tableau I). Presque 36,5 p. 100 des chèvres ont eu un intervalle mise bas - mise bas dépassant la moyenne, et seulement 8,8 p. 100 des chèvres ont enregistré un intervalle inférieur à 210 jours (figure 4 ). Ce long intervalle entre deux mises bas successives peut aussi s'expliquer par la mise à la reproduction tardive des chèvres pratiquée sur la station. Cet intervalle a été plus élevé que celui observé chez les chèvres Red Sokoto (9) et chez les chèvres locales coréennes (208 jours) (19). Il a été également supérieur à l'intervalle de 7,46 mois obtenu chez les éleveurs de la race Draa (8). En revanche, il a été similaire aux intervalles entre mises bas de 350 et 355 jours enregistrés respectivement chez les

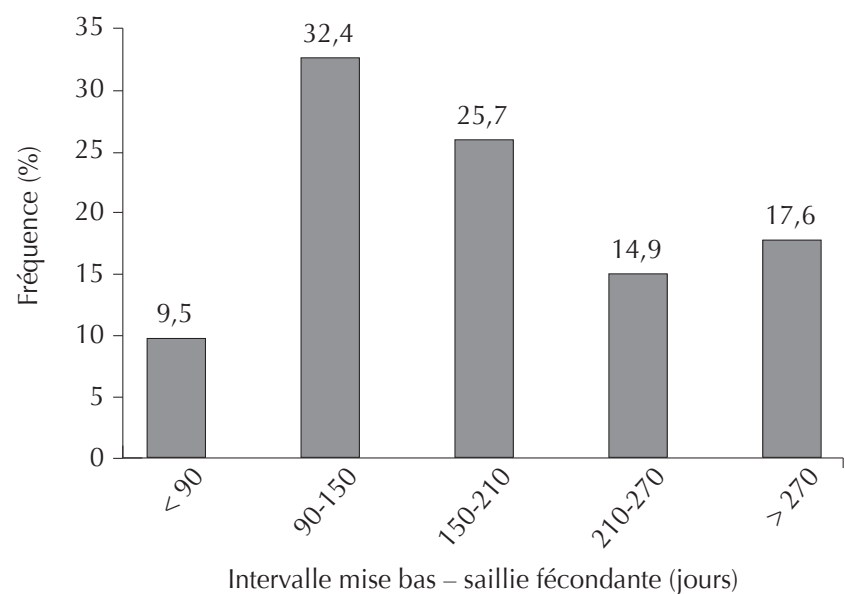

Figure 3 : distribution des fréquences de l'intervalle mise bas - saillie fécondante des chèvres Draa $(n=74)$.

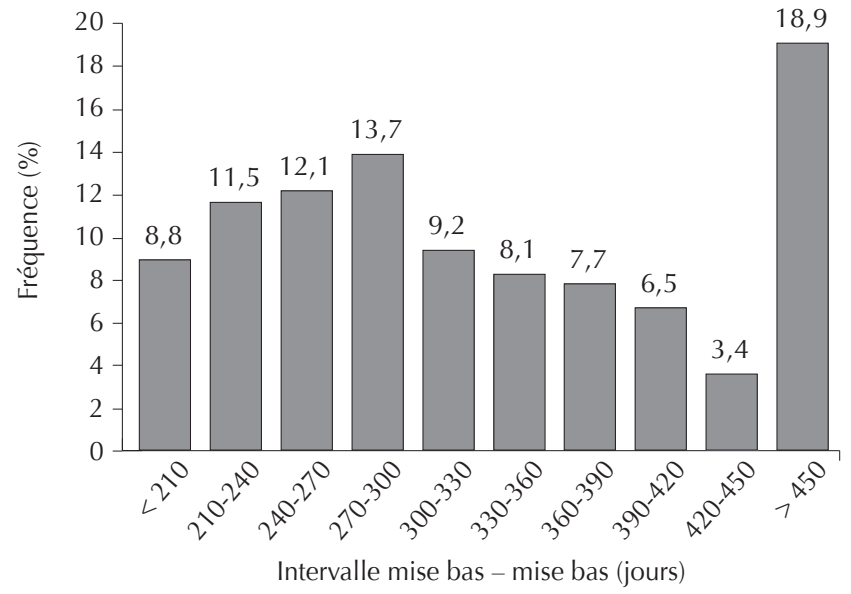

Figure 4 : distribution des fréquences de l'intervalle mise bas - mise bas des chèvres Draa $(n=555)$. 
chèvres de race Alpine et Anglo-Nubienne (6), et de 378 jours chez les chèvres de Maradi (14).

\section{Durée de gravidité}

La moyenne de la durée de gravidité des chèvres Draa a été de 153 jours, avec un écart-type de 1,34 jour (tableau I). La classe modale (67,9 p. 100) correspondait à la classe $152-154$ jours (figure 5). Comparée à la durée de gravidité des races Matou de Chine (150 jours) d'après Moaeen-Ud-Din et coll. (16), Boer (148,2 jours) d'après Greyling (7), et de trois races indiennes $(145,148$ et 152 jours) d'après Zeshmarani et coll. (20), celle de la Draa a semblé particulièrement longue. Cette longue durée de gravidité semble être une caractéristique génétique de la race Draa.

\section{Taille de la portée à la naissance}

La taille de la portée à la naissance des chèvres Draa a varié de 1 à 3 chevreaux avec une moyenne de 1,40. La figure 6 montre la distribution des portées simples, doubles et triples. La taille de la portée à la naissance des chèvres Draa est intéressante en ce qu'elle semble indiquer un bon potentiel génétique pour ce caractère. Selon une autre étude réalisée sur cette même race mais conduite selon un mode très intensif, les portées simples, doubles et triples ont représenté respectivement 36, 57 et 7 p. 100 (4). Par ailleurs, la

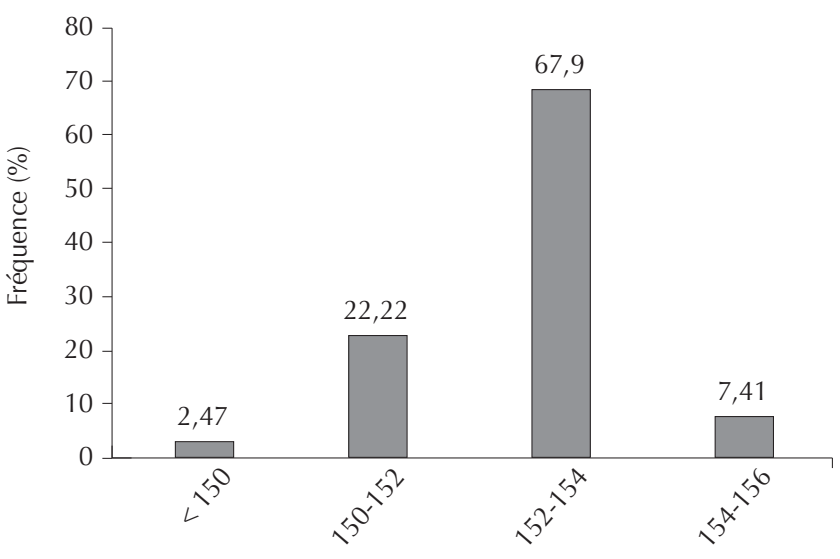

Durée de gravidité (jours)

Figure 5 : distribution des fréquences de la durée de gravidité des chèvres Draa $(n=79)$.

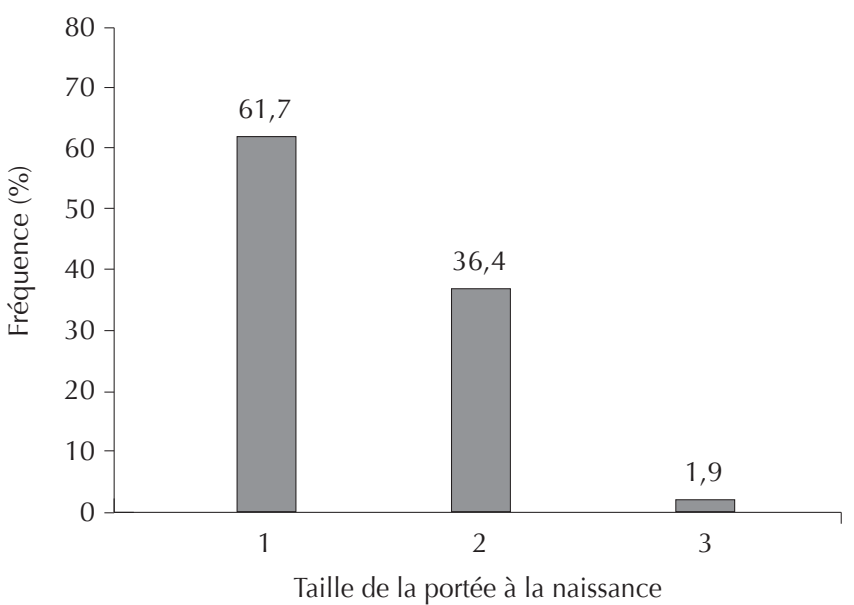

Figure 6 : distribution de fréquences de la taille de la portée à la naissance des chèvres Draa $(n=841)$. taille de la portée à la naissance des chèvres Draa a été légèrement supérieure à celle de 1,32 rapportée pour les chèvres de Maradi (14), mais légèrement inférieure à celle de 1,74 relevée sur des chèvres locales coréennes (19).

\section{Poids corporel à la mise bas}

Le poids corporel des chèvres Draa à la mise bas a été en moyenne de $31,3 \mathrm{~kg}$, avec un coefficient de variation de 20,4 p. 100 (tableau I). La figure 7 montre la distribution des fréquences de poids à la mise bas. Le faible poids corporel des chèvres Draa reflète les conditions climatiques sévères dans lesquelles cette race est élevée. Néanmoins, ce poids a été légèrement plus élevé que celui de 28,1 kg relevé chez des chèvres de Maradi (14).

\section{Durée de lactation}

La durée de lactation a été en moyenne de 133 jours, avec un coefficient de variation de 33,9 p. 100 (tableau I). Cette durée a varié de 20 à 317 jours. Les durées de lactation inférieures à trois mois ont représenté 13,4 p. 100 des cas, celles comprises entre trois et cinq mois 57,6 p. 100, et celles supérieures à neuf mois 16 p. 100 (figure 8). Cette durée de lactation a été plus courte que celle de 150 jours observée chez les chèvres de la même race (4) et chez les races laitières spécialisées (15).

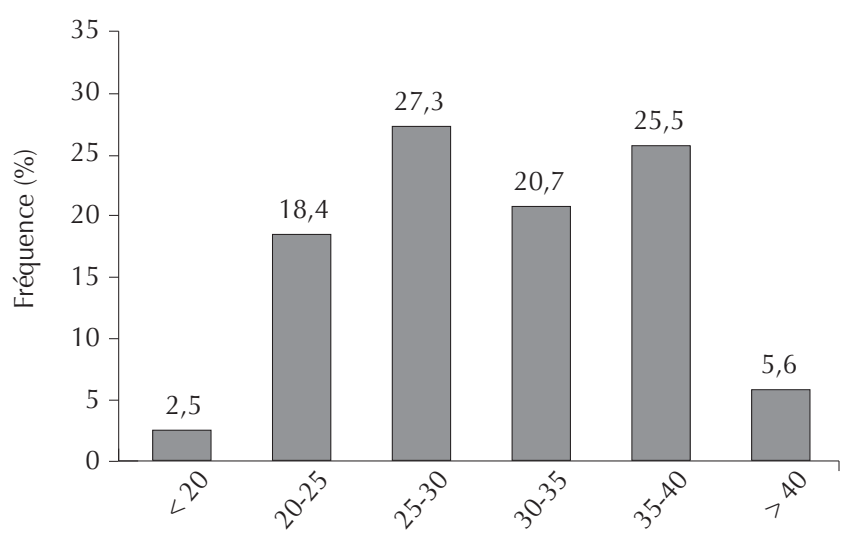

Poids à la mise bas (kg)

Figure 7 : distribution des fréquences du poids vif à la mise bas des chèvres Draa $(n=792)$.

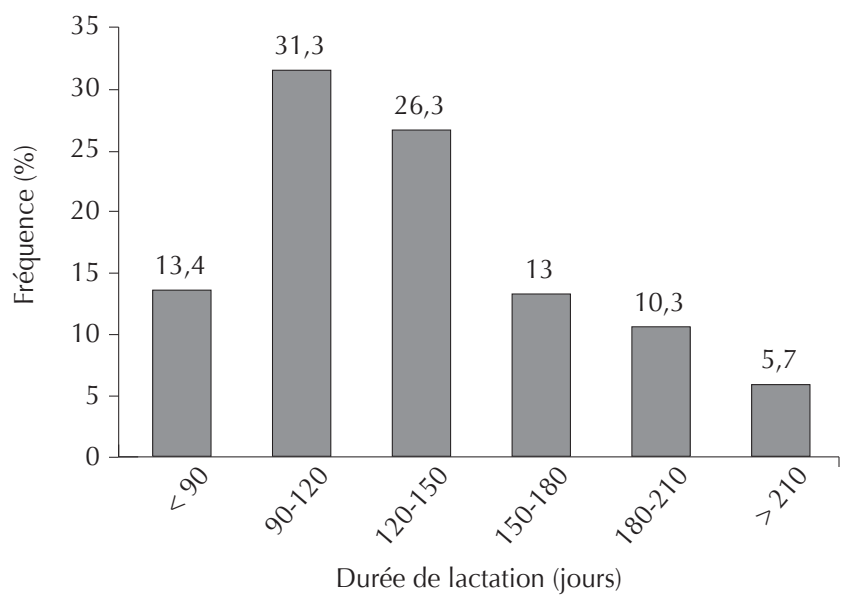

Figure 8 : distribution des fréquences de la durée de lactation des chèvres Draa $(n=754)$. 


\section{Quantité de lait par lactation totale}

La quantité de lait par lactation totale des chèvres Draa a été en moyenne de $61,3 \mathrm{~kg}$, avec un coefficient de variation de 38,8 p. 100 (tableau I). En outre, 14,2 p. 100 des quantités de lait ont été inférieures à $40 \mathrm{~kg}, 69,2 \mathrm{p} .100$ ont été comprises entre 40 et $80 \mathrm{~kg}$, et 16,4 p. 100 ont été supérieures à $80 \mathrm{~kg}$. La valeur maximale enregistrée a été de $191 \mathrm{~kg}$ (figure 9). La faiblesse de la production laitière des chèvres Draa dans la présente étude peut s'expliquer soit par son faible potentiel génétique, malgré une réputation de bonne laitière, soit par les conditions de la station qui n'ont pas permis aux chèvres d'extérioriser pleinement leur potentiel de production.

La production laitière par lactation totale des chèvres Draa a été en deçà de celle rapportée $(142 \mathrm{~kg})$ chez la même race élevée selon un mode de conduite très intensif (4). Comparée aux races laitières spécialisées dans le monde, la production laitière des chèvres Draa a été très inférieure à celle des chèvres Murciano-Granadina, qui est de $334 \mathrm{~kg}$ (1), et Saanen, qui varie de 667 à 754 kg (11).

\section{Production laitière journalière}

La production laitière journalière des chèvres Draa au cours de toute la lactation a été en moyenne de $0,46 \mathrm{~kg}$ avec un coefficient de variation de 56,5 p. 100 (tableau I). La production maximale a été de $1,9 \mathrm{~kg}$.

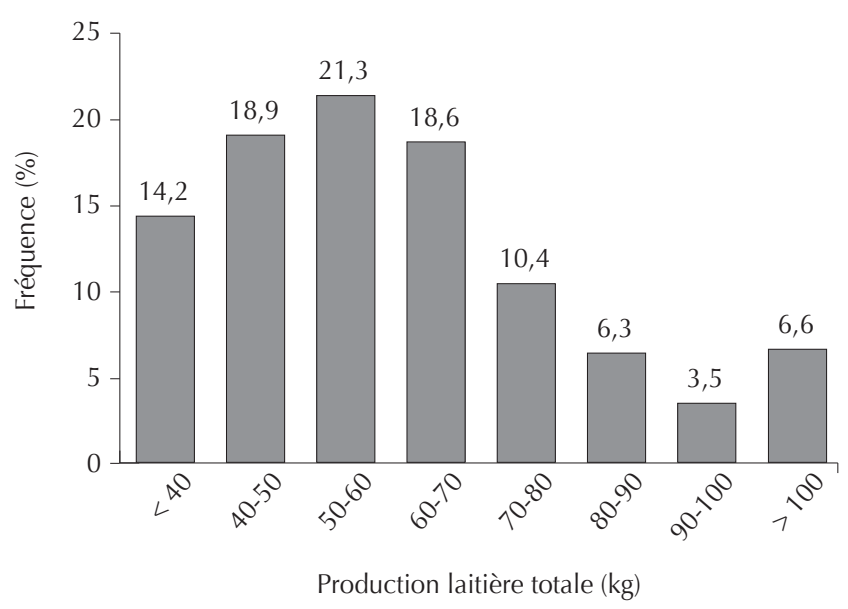

Figure 9 : distribution des fréquences de la production laitière totale des chèvres Draa $(n=709)$.
La figure 10 montre que la production laitière journalière a fluctué en dents de scie autour de $0,7 \mathrm{~kg}$ jusqu' au quarante-cinquième jour de lactation, puis elle a diminué progressivement pour atteindre une production de $0,1 \mathrm{~kg}$ vers le cent unième jour.

La production laitière journalière a été inférieure à celle de $0,77 \mathrm{~kg}$ enregistrée chez la même race (8) et à la celle de 3,44 kg observée chez les chèvres Alpines élevées dans les conditions marocaines (12). Chez les chèvres primipares de race Murciano-Granadina, la production laitière moyenne a augmenté de $1,59 \mathrm{~kg} / \mathrm{jour}$ à la deuxième semaine de lactation jusqu'au pic de production de $1,62 \mathrm{~kg} /$ jour atteint à la sixième semaine, puis a diminué jusqu'à $1,2 \mathrm{~kg} / \mathrm{j}$ à la dix-huitième semaine (5).

\section{CONCLUSION}

L'analyse des performances de reproduction et de production laitière des chèvres Draa a permis de préciser leurs aptitudes dans ces domaines. Elle a également permis de détecter une grande variabilité au sein de la race pour différents caractères qui pourrait être mise à profit à travers des programmes de sélection, sous réserve de mesurer ultérieurement la variabilité génétique. Néanmoins, la faible production laitière enregistrée dans la présente étude mériterait des études complémentaires.

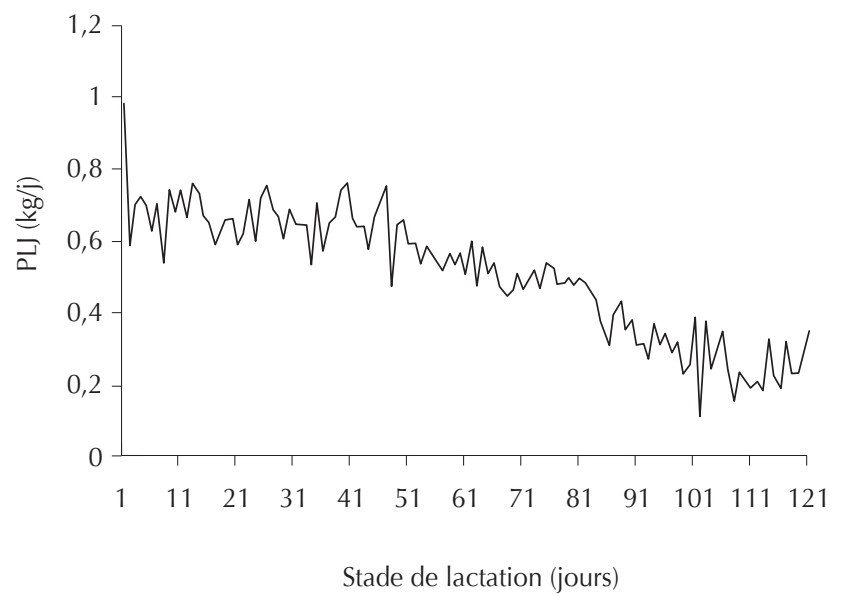

Figure 10 : évolution de la production laitière journalière $(P L J)$ en fonction du stade de lactation des chèvres Draa.

\section{BIBLIOGRAPHIE}

1. ANALLA M., JIMENEZ-GAMERO I., MUNOZ-SERRANO A., SERRADILLA J.M., FALAGANS A., 1996. Estimation of genetic parameters for milk yield and fat and protein contents of milk from Murciano-Granadina goats. J. Dairy Sci., 79: 1835-1898.

2. BOUJENANE I., 2008. Eléments de réflexion sur I'amélioration génétique des caprins au Maroc. Eleveur, 16 : 13-16.

3. BOURFIA M., 1989. Caractéristiques distinctives des populations caprines marocaines. In : Proc. Sémin. Association nationale pour la production animale. Ouarzazate, Maroc, ministère de l'Agriculture et de la Réforme agraire, p. 88-89.

4. EZZAHIRI A., BEN LAKHAL M., 1989. Performances de la chèvre D’man élevée en station au Maroc. Maghreb vét., 4 : 30.
5. FERNANDEZ C., SANCHEZ A., GARCES C., RUBERT-ALEMAN J., DIAZ J.R., 2000. Courbe de lactation chez la chèvre Murciano-Granadina primipare. Renc. Rech. Ruminants, $7: 136$.

6. GILL G.S., DEV D.S., 1972. Performance of two exotic breeds of goats under Indian conditions. Indian J. Anim. Prod., 3: 173.

7. GREYLING J.P.C., 2000. Reproduction traits in the Boer goat doe. Small Rumin. Res., 36: 171-177.

8. HACHI A., 1990. La chèvre D'man : Contribution à l'étude des caractéristiques de reproduction. Thèse Doct. vét., Institut agronomique et vétérinaire Hassan II, Rabat, Maroc.

9. HAUMESSER J.B., 1975. Quelques aspects de la reproduction chez la chèvre rousse de Maradi. Comparaison avec d'autres races tropicales ou subtropicales. Revue Elev. Méd. vét. Pays trop., 28 : 225-234. 
10. ICAR, 2009. International agreement of recording practices. In: ICAR General Assembly, Niagara Falls, USA, 18 June 2008.

11. KENNEDY B.W., FINLEY C.M., BRADFORD G.E., 1982. Phenotypic and genetic relationships between reproduction and milk production in dairy goats. J. Dairy Sci., 65: 2373-2383.

12. LAKHDAR A., 2004. Evaluation génétique des chèvres laitières Alpines du domaine Douiet. Mém. $3^{\mathrm{e}}$ Cycle Ing. Agron., ENA, Meknès, Maroc.

13. MAPM, 2008. Elevage en chiffres 2007. Rabat, Maroc, direction de I'Elevage, ministère de l'Agriculture et de la Pêche maritime.

14. MARICHATOU H., MAMANE L., BANOIN M., BARIL G., 2002. Performances zootechniques des caprins au Niger : étude comparative de la chèvre rousse de Maradi et de la chèvre à robe noire dans la zone de Maradi. Revue Elev. Méd. vét. Pays trop., 55 : 79-84.

15. MAVROGENIS A.P., PAPACHRISTOFOROU C., LYSANDRIDES P., ROUSHIAS A., 1989. Environmental and genetics effects on udder characteristics and milk production in Damascus goats. Small Rumin Res., 2: 333-343.

\section{Summary}

Boujenane I., Lichir N., El Hazzab A. Reproduction and milk production performances of Draa goats in Morocco

The main objective of this study was a contribution to the knowledge of Draa goats. The study concerned the analysis of reproduction and milk production performances of Draa goats at Skoura research station from 1989 to 2001. Age at first kidding, gestation period, kidding interval, and kidding to conception interval averaged 25.5 months, 153 days, 157 days and 206 days, respectively. The lactation period was 133 days. Daily milk production and total milk production were 0.46 and $61.3 \mathrm{~kg}$, respectively. It was concluded that it would be interesting to make use of the genetic variability present in the breed for selection purposes.

Keywords: Goat - Draa nannigoat - Reproduction - Milk production - Parturition interval - Lactation duration Morocco.
16. MOAEEN-UD-DIN M., YAND L.G., CHEN S.L., ZHANG Z.R., XIAO J.Z., WEN Q.Y., DAI M., 2008. Reproductive performance of Matou goat under sub-tropical monsoonal climate of Central China. Trop. Anim. Health Prod., 40: 17-23.

17. NEERU, SINGH D., KUMAR P., GOEL A.K., 2003. Factors affecting postpartum oestrus interval in goats. Indian J. Anim. Sci., 73: 408-409.

18. SAS, 1996. SAS/STAT, User's guide. Cary, NC, USA, SAS Institute.

19. SONG H.B., JO I.H., SOL H.S., 2006. Reproductive performance of Korean native goats under natural and intensive conditions. Small Rumin. Res., 65: 284-287.

20. ZESHMARANI S., DHARA K.C., SAMANTA A.K., SAMANTA R., MAJUMDER S.C., 2007. Reproductive performance of goats in Eastern and North-Eastern India. Livest. Res. Rural Dev., 19.

Accepté le 13.06.2012

\section{Resumen}

Boujenane I., Lichir N., El Hazzab A. Rendimientos de reproducción y de producción lechera de las cabras Draa en Marruecos

El objetivo principal del presente estudio fue una contribución al conocimiento de las cabras de raza Draa. El estudio se centró en el análisis de los rendimientos de reproducción y de producción lechera de las cabras Draa de la estación de Skoura, entre 1989 y 2001. La edad al primer parto fue en promedio de 25,5 meses. La duración promedio de la preñez fue de 153 días. El intervalo entre dos partos y el intervalo parto-monta fecundante fueron respectivamente de 157 y de 206 días. La duración de la lactación fue de 133 días. La producción lechera fue de 0,46 kg/día y de $61,3 \mathrm{~kg}$ por lactación total. Se concluye que sería interesante, con objetivos de selección, explotar la variabilidad genética que existe en el seno de la raza.

Palabras clave: Caprino - Cabra Draa - Reproducción Producción lechera - Intervalo entre partos - Duración de la lactancia - Marruecos. 